\title{
LEARNING THE ROPES: \\ PULMONARY HYPERTENSION
}

\section{Pulmonary hypertension clinical presentation and relevance}
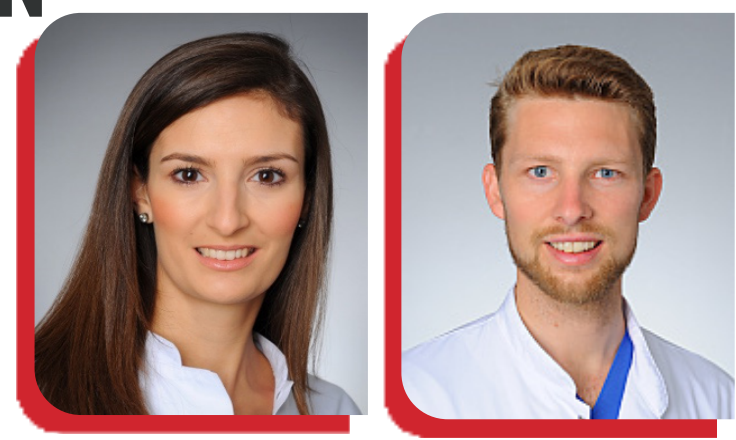

\section{JOANA ADLER, MAX WISSMÜLLER, \& STEPHAN ROSENKRANZ}

Clinic III for Internal Medicine, Heart Center at the University of Cologne, and Cologne Cardiovascular Research Center (CCRC), Cologne, Germany

Pulmonary hypertension (PH) is a hemodynamic condition affecting approximately $1 \%$ of the world population, and the prevalence in adults $>65$ years is about $10 \%{ }^{1}$. Incidence and prevalence are related to the underlying cause, $\mathrm{PH}$ associated with left heart diseases and/or chronic lung diseases representing the most common forms ${ }^{1}$.

Based on the underlying etiology and clinical presentation, the updated clinical classification of $\mathrm{PH}$ categorizes five groups ${ }^{2}: 1$. Pulmonary arterial hypertension (PAH); 2. Pulmonary hypertension due to left heart disease; 3. Pulmonary hypertension due to lung disease and/ or hypoxia; 4. Pulmonary hypertension due to pulmonary artery obstruction; 5. Pulmonary hypertension with unclear and/or multifactorial mechanisms (Table 1). Despite the differing etiology, all forms of $\mathrm{PH}$ are linked to progressive clinical symptoms and associated with excess mortality ${ }^{3}$. In fact, even moderate elevations of pulmonary artery pressure (PAP) and pulmonary vascular resistance (PVR) are associated with increased mortality ${ }^{4,5}$.

PAH is a relatively rare disease of the small pulmonary resistance vessels that may affect younger individuals, and - if left untreated leads to right heart failure and death ${ }^{6}$. As the disease is slowly progressing, clinical signs and symptoms usually develop gradually over time and at initial stages are often unspecific, PAH is usually diagnosed with a significant delay. The clinical picture resembles exertional dyspnea, reduced exercise capacity, bendopnoe, and fatigue. Advanced stages are characterized by signs of right heart failure such as venous congestion, peripheral edema, and ascites, along with states of exhaustion and syncope (during or after physical effort) ${ }^{3,6}$. Progressive right ventricular dysfunction may lead to episodes of cardiac decompensation that are associated with a high mortality risk.

Impaired right ventricular function results in backward failure and systemic congestion, but also in diminished left ventricular filling and reduced systemic output, at rest and during exercise. The combination of the two triggers systemic inflammatory reactions and inter-organ crosstalk, affecting multiple organ systems (Figure 1) ${ }^{7}$. These systemic consequences of $\mathrm{P}(\mathrm{A}) \mathrm{H}$ place additional burden on patients and may represent treatable traits in addition to targeted therapy of $\mathrm{PH}$ and underlying causes ${ }^{7}$.

The diagnostic work-up in patients with suspected $\mathrm{PH}$ follows a diagnostic algorithm, aiming at establishing a precise diagnosis and classification of $\mathrm{PH}^{8}$. This is of utmost importance, as the 
therapeutic consequences differ substantially between the distinct forms of $\mathrm{PH}$. In addition to physical examination, non-invasive tools and findings include ECG-signs of right heart strain, reduced exercise capacity (assessed by cardiopulmonary exercise testing [CPET] and the 6 minute walk distance [6MWD]), and elevated $\mathrm{N}$-terminal fragment of brain natriuretic peptide (NTproBNP) serum levels ${ }^{8}$. Echocardiography is of particular importance, as it may show elevations of the estimated systolic pulmonary arterial pressure (PASP), and pathological changes of right heart dimensions and function ${ }^{8}$. More recently, cardiac MRI has also been established to accurately assess RV size, morphology and function, and allows non-invasive measurement of stroke volume, cardiac output (CO) as well as pulmonary arterial flow. Nevertheless, the diagnosis of PH can only be confirmed by right heart catheterization (RHC). PH is defined by an elevation of the mean pulmonary arterial pressure (mPAP) $\geq 25$ $\mathrm{mmHg}$ at rest, although lowering the threshold to $>20 \mathrm{mmHg}$ has recently been proposed ${ }^{2}$. The hemodynamic definition sub-classifies $\mathrm{PH}$ into a pre- and post-capillary form, depending on the pulmonary arterial wedge pressure (PAWP). The hemodynamic profile in PAH is characterized by pre-capillary $\mathrm{PH}$, defined by a PAPm $\geq 25 \mathrm{mmHg}$, a PAWP $\leq 15 \mathrm{mmHg}$, and a PVR $>3 \mathrm{WU}^{3,8}$.

Among patients with $\mathrm{PAH}$, there appear to be distinct clinical phenotypes. While idiopathic PAH was originally recognized as a disease of younger patients with a female predominance, who had no or few comorbidities, recent registry analyses have shown that PAH is now increasingly diagnosed in elderly patients that may have multiple comorbidities. Those patients meet the hemodynamic criteria of PAH (pre-capillary PH), but disease characteristics, pathophysiology, and the distinction from group $2 \mathrm{PH}$ are more complex and challenging. In addition, response to targeted therapy and the tolerability of PAH drugs are less well studied. To better describe the distinct phenotypes, the terms "typical" or "classical" $\mathrm{PAH}$ (younger patients with few comorbidities) and "atypical PAH" or "PAH with comorbidities" (elderly patients with multiple comorbidities) have been introduced ${ }^{9}$. Although the evidence for the safety and efficacy of PAH medications was mainly derived from patients with "classical PAH", recent analyses indicated that the group of "PAH with comorbidities" also benefits from targeted therapy ${ }^{10,11}$.

Consistent with the distinction of PAH phenotypes, a recent cluster analysis of patients with idiopathic PAH from the COMPERA registry which was based on age, sex, DLCO (diffusing capacity of the lungs for carbon monoxide), smoking status and the presence of comorbidities (obesity, hypertension, CHD, diabetes mellitus) identified distinct patient clusters, which differed in clinical presentation, response to therapy, and survival ${ }^{12}$. Specifically, a cluster of younger age and without comorbidities had a better response to PAH treatment and better outcome when compared to clusters of elderly patients with comorbidities, lower DLCO, and smoking history. However, there were also differences in the treatment patterns with less combination therapies in the latter groups.

In order to predict outcome and inform treatment decisions, the current ESC/ERS guidelines recommend a risk assessment strategy for patients with $\mathrm{PAH}^{8}$. Based on a multimodal approach considering various clinical measures, patients are categorized as "low risk", "intermediate risk", or "high risk". This strategy was recently validated independently in 3 large registries, which consistently proved its validity both at the time of diagnosis and during follow-up. According to these studies, risk assessment in patients with PAH provides accurate mortality estimates, with mortality rates in low, intermediate, and high risk patients of $<5 \%, 5-10 \%$, and $>10 \%$, respectively. Furthermore, a simplified risk assessment strategy quantifying the number of low-risk criteria accurately predicted transplant-free survival, and patients with key non-invasive variables (WHO-FC, 6MWD, NTproBNP) in the low-risk category had an excellent 5-year survival. Hence, an important treatment goal is to achieve a low-risk profile, i.e. a high number of low-risk criteria.

It should be acknowledged, however, that risk assessment has been validated primarily for patients with "classical PAH", whereas it has to be further evaluated in "PAH with comorbidities". Moreover, the efficacy and safety of targeted PAH therapies, as well as treatment strategies, must be further assessed in the latter group in randomized controlled trials. 
Figure 1. Systemic consequences of $\mathrm{P}(\mathrm{A}) \mathrm{H}$ and right-sided heart failure: Interdependent mechanisms, systemic inflammation, and interorgan cross-talk ${ }^{7}$ Republished with permission from AHA.

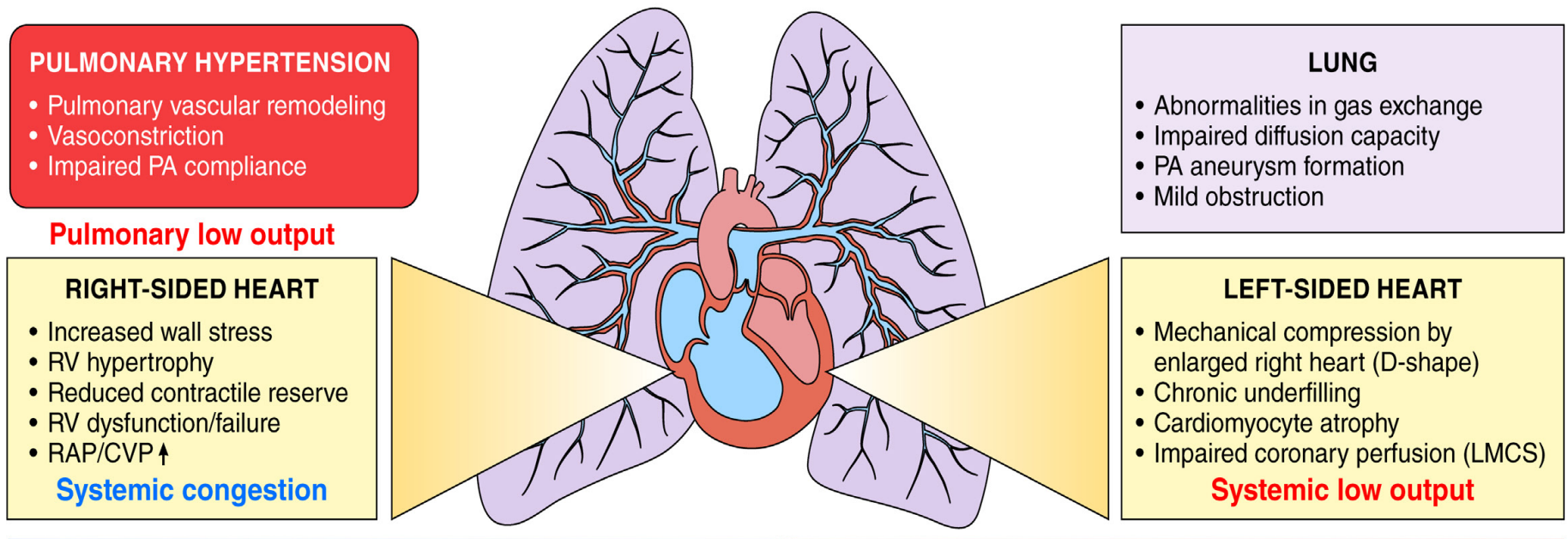

\begin{tabular}{|c|c|c|c|c|}
\hline $\begin{array}{l}\text { Activation of } \\
\text { venous endothelium }\end{array}$ & $\begin{array}{l}\text { Sinusoidal congestion } \\
\text { Endothelial activation }\end{array}$ & $\begin{array}{c}\text { "Leaky bowel syndrome" } \\
\text { bacterial translocation/ } \\
\text { endotoxin } \uparrow\end{array}$ & $\begin{array}{c}\text { Cerebral tissue oxygenation } \\
\text { Neurohormonal } \\
\text { activation }\end{array}$ & $\begin{array}{l}\text { Hypoperfusion/hypoxia } \\
\text { underutilization }\end{array}$ \\
\hline \multicolumn{5}{|c|}{$\begin{array}{l}\text { Soluble / circulating pro-inflammatory mediators (chemokines/cytokines) } \\
\text { (e.g. TIMP-2, IGFBP-7, NGAL, L-FABP, KIM-1, } \alpha \text {-GST, } \pi \text {-GST, TNF } \alpha \text {, IL-1 } \beta, \text { L-6, IL-10, TLR,...) }\end{array}$} \\
\hline Kidneys & Liver & GI Tract & Brain & Skeletal muscle \\
\hline
\end{tabular}


Table 1. Updated clinical classification of pulmonary hypertension (PH); according to the 6th World Symposium on Pulmonary Hypertension²

\begin{tabular}{|l|}
\hline 1. Pulmonary arterial hypertension (PAH) \\
1.1 Idiopathic PAH \\
1.3 Drug- and toxin-induced PAH \\
1.4 PAH associated with: \\
1.4.1 Connective tissue disease \\
1.4.2 HIV infection \\
1.4.3 Portal hypertension \\
1.4.4 Congenital heart disease \\
1.4.5 Schistosomiasis| \\
1.5 PAH long-term responders to calcium channel blockers \\
1.6 PAH with overt features of venous/capillaries (PVOD/PCH) involvement \\
1.7 Persistent PH of the newborn syndrome \\
\hline 2. PH due to left heart disease \\
2.1 PH due to heart failure with preserved LVEF \\
2.2 PH due to heart failure with reduced LVEF \\
2.3 Valvular heart disease \\
2.4 Congenital/acquired cardiovascular conditions leading to post-capillary PH
\end{tabular}

References

1.Hoeper MM, , et al. (2016) A global view of pulmonary hypertension. The Lancet Respiratory Medicine 4: 306-322.

2.Simonneau G, et al.R (2019) Haemodynamic definitions and updated clinical classification of pulmonary hypertension. Eur Respir J 53. doi: 10.1183/13993003.01913-2018

3. Rosenkranz S, et al.Left ventricular heart failure and pulmonary hypertension. Eur Heart J 2016; 37: 942-954.

4.Maron BA, et al.Association of borderline pulmonary hypertension with mortality and hospitalization in a large patient cohort: Insights from the Veterans Affairs clinical assessment, reporting, and tracking program. Circulation 2016; 133: 1240-1248.

5.Maron BA, et al. Pulmonary vascular resistance and clinical outcomes in patients with pulmonary hypertension: a retrospective cohort study. Lancet Respir Med 2020; 8: 873-884.

6. Humbert $M$, et al. Pathology and pathobiology of pulmonary hypertension: state of the art and research perspectives. Eur Respir J 2019 Jan 24;53(1):1801887.

\author{
3. PH due to lung diseases and/or hypoxia \\ 3.1 Obstructive lung disease \\ 3.2 Restrictive lung disease \\ 3.3 Other lung disease with mixed restrictive/obstructive pattern \\ 3.4 Hypoxia without lung disease \\ 3.5 Developmental lung disorders
}

\section{PH due to pulmonary artery obstructions}

4.1 Chronic thromboembolic $\mathrm{PH}$

4.2 Other pulmonary artery obstructions

\author{
5. $\mathrm{PH}$ with unclear and/or multifactorial mechanisms \\ 5.1 Haematological disorders \\ 5.2 Systemic and metabolic disorders \\ 5.3 Others \\ 5.4 Complex congenital heart disease
}

7.Rosenkranz S, et al. Systemic consequences of pulmonary hypertension and right-sided heart failure. Circulation 2020; 141: 678-692.

8.Galiè N, et al. (2016) 2015 ESC/ERS Guidelines for the diagnosis and treatment of pulmonary hypertension: The Joint Task Force for the Diagnosis and Treatment of Pulmonary Hypertension of the European Society of Cardiology (ESC) and the European Respiratory Society (ERS): Endorsed by: Association for European Paediatric and Congenital Cardiology (AEPC), International Society for Heart and Lung Transplantation (ISHLT). Eur Heart J $37: 67-119$

9.Hoeper MM, et al. Targeted therapy of pulmonary arterial hypertension: Updated recommendations of the Cologne Consensus Conference 2018. Int J Cardiol 2018; 272S: 37-45.

10. McLaughlin W, et al. Patients with pulmonary arterial hypertension with and without cardiovascular risk factors: Results from the AMBITION trial. J Heart Lung Transplant 2019; 38: 1286-1295

Full reference list

Stephan Rosenkranz - stephan.rosenkranz@uk-koeln.de 